\title{
Analysis of Plasma Orexin Levels in Pedıatric Migraine Patients: A Prospective Controlled Clinical Study
}

\author{
Hulya Ince ${ }^{1(\mathbb{D})}$ Omer Faruk Aydin ${ }^{2(\mathbb{I D})}$ Hasan Alacam $^{3(\mathrm{ID})}$ Erhan Cetin Cetinoglu ${ }^{4(\mathrm{ID})}$ \\ ${ }^{1}$ Department of Pediatrics, Division of Pediatric Neurology, Private Medical Park Samsun, Hospital, Samsun, Turkey \\ ${ }^{2}$ Department of Pediatrics, Division of Pediatric Neurology, Ondokuz Mayıs University, Samsun, Turkey \\ ${ }^{3}$ Department of Biochemistry Ondokuz Mayıs University, Samsun, Turkey \\ ${ }^{4}$ Department of Community Health, Ondokuz Mayıs University, Samsun, Turkey
}

Copyright@ Author(s) - Available online at https://dergipark.org.tr/en/pub/mbsjohs Content of this journal is licensed under a Creative Commons Attribution-NonCommercial 4.0 International License,

Received: 03 April 2021, Accepted: 19 August 2021, Published online: 31. August 2021

(C) Ordu University Institute of Health Sciences, Turkey, 2021

\begin{abstract}
Objective: Migraine is the most common primary headache disorder in children. However, its pathogenetic mechanisms are not fully understood. Researchers focused on Orexin A (a neuropeptide with anti-nociceptive effects) and Orexin B (a neuropeptide with pro-nociceptive effects), but the literature is scarce in terms of studies investigating the plasma levels of these neuropeptides in pediatric migraine patients. We aimed to compare the plasma levels of orexins between pediatric migraine patients and healthy controls

Methods: Children aged between 5 and 18 who were under diagnostic evaluation for and diagnosed with migraine in Ondokuz May1s University, Department of Pediatric Neurology between December 2018 and December 2019, constituted the target population. All study group patients (Group 1) were diagnosed with migraine according to IHS 2004 criteria. The control group (Group 2) consisted of healthy children. Data including age, gender, and presence or absence of aura were recorded. Two blood samples were taken from the patients in Group 1. The first sample was withdrawn during a non-attack period, and the second sample was withdrawn during the initial migraine attack. Only one blood sampling was done in the control group. Plasma Orexin A and Orexin B levels were analyzed by radioimmunoassay and compared between Group 1 and Group 2 during non-attack and attack periods. Also, intra-group comparative analyses were performed. Non-parametric tests were used for statistical analysis.

Results: This study included 98 patients, 52 children with migraine (Group 1), and 46 healthy children

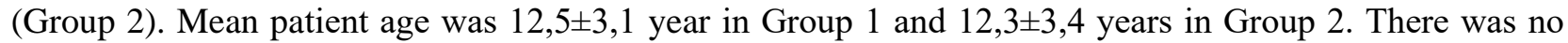
difference between patient groups in terms of gender $(\mathrm{p}=0,103)$ and age $(\mathrm{p}=0,734)$. Plasma Orexin A levels of the migraine patients were higher than control group participants during the non-attack period, while Orexin B levels of the migraine patients were higher than migraine patients during the attack period. The mean plasma Orexin A level was significantly higher during the non-attack period than the attack period $(\mathrm{p}=0,002)$. The mean plasma Orexin B level was significantly higher during the attack than in the non-attack period $(\mathrm{p}=0,002)$. The presence of aura did not impact plasma orexin levels during both attack and non-attack periods.

Conclusions: The plasma level of Orexin A is elevated in migraine patients, probably as a response to nociceptive signals, and migraine attack is associated with elevated plasma Orexin B levels. Targeting the orexinergic system seems like a reasonable approach to improving the treatment of migraine disease.

Key words: migraine, arexin, pediatric, headache, treatment, children
\end{abstract}

Suggested Citation: Ince H, Aydin O F, Alacam H, Cetinoglu E C. Analysis of Plasma Orexin Levels in Pediatric Migraine Patients: A Prospective Controlled Clinical Study. Mid Blac Sea Journal of Health Sci, 2021; 7(2):269-275

\section{Address for correspondence/reprints:}

Hulya Ince
Telephone number: +90 (532) 1354070

E-mail: drhulyaince@yahoo.com 


\section{Introduction}

The International Headache Society (IHS) defines migraine as recurrent, unilateral, pulsating headache of moderate or severe intensity which lasts for 4 to 72 hours $(1,2)$. It is estimated that migraine affects 3 $10 \%$ of children (3-5). Migraine is the most common primary headache disorder in children and adolescents, and approximately $18 \%$ of cases encountered in pediatric emergency rooms are migraine-related $(6,7)$.

Tajti et al. reported that the trigeminovascular system (TVS), which includes trigeminal ganglion, meningeal vasculature, thalamus, and somatosensory cortex, took an important role in the pathogenesis of migraine (8). They also denoted that activation of TVS and neurogenic dural vasodilatation could be involved in the pathogenesis of migraine, and orexins were the neuropeptides involved in the central and peripheral sensitization of the trigeminal system. It is known that orexins are responsible for the regulation of autonomic functions and the sleep-wake cycle (8). Since migraine is related to autonomic dysfunction and sleep disorders such as insomnia; these authors noted that further clinical trials were needed to identify better the role of orexins in the pathogenesis of migraine (8).

Despite the questions regarding the essential role of orexins (i.e., orexin A and orexin B) in migraine pathogenesis, the literature is scarce regarding clinical studies analyzing the levels of these neuropeptides in migraine patients (8-10) A few numbers of clinical trials conducted with adult patients gave contradictory results and -to the best of our knowledge- there have been no published reports assessing the levels of these neuropeptides in pediatric migraine patients (8-10).

This study aimed to investigate the plasma levels of orexin A (OrxA) and orexin B (OrxB) in pediatric migraine patients, expecting our findings to contribute to the ongoing research regarding the pathogenesis of pediatric migraine.

\section{Methods}

This prospective study was approved by the ethical review committee of Ondokuz May1s University (OMU) Hospital (2018/174). Pediatric patients under diagnostic evaluation for and subsequently diagnosed with migraine in OMU Children's Hospital, Department of Pediatric Neurology, between December 2018 and December 2019 constituted this study's target population. All study group patients were under diagnostic management with the preliminary diagnosis of migraine and had a migraine attack during this diagnostic evaluation period before initiating a prophylactic migraine treatment. All these patients were later diagnosed with migraine as per IHS 2004 criteria.1 For the control group, participants were selected among children who presented to Healthy Children's Outpatient Clinic of the same hospital during the same time frame.

Patients whose parents approved their participation were assigned to this study. All parents gave their written consent before the participation of their children in this study. Patients who were younger than five or older than 18 patients diagnosed with a migraine disease before the study period and prescribed prophylactic treatment were omitted. Patients who have another type of chronic headache syndrome or any other endocrinologic, cardiovascular, or neurologic disease that can cause autonomic dysfunction were also excluded. The control group consisted of completely healthy children aged between 5 and 18 without a family history of migraine. All authors had full access to all study patient data during the study period.

All study group (Group 1) and control group (Group 2) participants underwent a thorough neurological examination. All study participants' demographic data and migraine-related data, including age, gender, presence, or absence of aura, were recorded. Two fasting blood samples were withdrawn from the patients in Group 1. The first blood sample was withdrawn during the initial outpatient encounter while the patient was in a nonattack period and under diagnostic management with the preliminary migraine diagnosis. The second blood sample was withdrawn during the first migraine attack of these patients after their first presentation to the pediatric neurology outpatient clinic and before initiation of prophylactic treatment. Both blood samples were taken from antecubital veins in the presence of ethylenediaminetetraacetic acid (EDTA; $1 \mathrm{mg} / \mathrm{ml}$ ), and each one was 3-4 milliliters in volume. These samples were immediately centrifuged at 3000 $\mathrm{g}$ for 10 minutes, and the plasma was kept in Eppendorf tubes and stored at $-80{ }^{\circ} \mathrm{C}$ until orexin level analysis. Plasma orexin levels were analyzed by radioimmunoassay (Peninsula Lab, San Carlos, CA, US) method following extraction using cartridges (Waters Associates, Milford, MA, US). The OrxA and OrxB kits were able to measure orexin levels as low as $10 \mathrm{pg} / \mathrm{ml}$. Intra-assay coefficients of variation were $5 \%$ and $5.3 \%$ for OrxA and OrxB, respectively.

Only one blood sampling was done in the control group using the same approach, and plasma orexin levels were analyzed using the same method. 
Plasma OrxA and OrxB levels were compared between Group 1 (migraine patients) and Group 2 (healthy controls) during non-attack and attack periods in Group 1. Also, intra-group comparative analyses were performed in Group 1 to compare plasma OrxA and OrxB levels during non-attack and attack periods and investigate the potential impact of aura on these levels.

\section{Statistical analysis}

Statistical analysis was performed by Statistical Package for Social Sciences (SPSS) 16.0 software. The results were given as means and standard deviations. Non-parametric tests, including the Mann-Whitney U test, Fisher's exact test, chi-square test, and Kruskal-Wallis test, were used to compare the groups. The $\mathrm{p}$ value was considered significant when it was lower than 0,05 .

\section{Results}

The entire study cohort included 98 participants; 52 children diagnosed with migraine (Group 1) and 46 healthy controls (Group 2). There were 30 girls and 22 boys in Group 1, while there were 18 girls and 28 boys in Group 2. Mean patient age was 12,5 $\pm 3,1$ year in Group 1 and 12,3 3,4 years in Group 2. There was no difference between the two groups in terms of gender distribution $(p=0,103)$ and age $(p=0,734)$. Plasma OrxA and OrxB levels of the patients in Group 1 during non-attack and attack periods and control group participants are displayed in Table 1.

The mean plasma OrxA level of the patients in Group 1 analyzed during the attack period was
$639,4 \pm 334,1[26,0-1106,4] \mathrm{pg} / \mathrm{ml}$. Comparison of this level with the mean plasma OrxA level of Group 2 $(503,6 \pm 322 \mathrm{pg} / \mathrm{ml}) \quad[19,4-990,3]$ revealed a statistically significant difference $(p=0,014)$. The mean plasma OrxB level of the patients in Group 1 analyzed during the attack period was $(1,7 \pm 1,5)[1,1$ 12] $\mathrm{pg} / \mathrm{ml})$. Comparison of this result with the mean plasma OrxB level of Group $2(1,3 \pm 0,3 \mathrm{pg} / \mathrm{ml})[1,1$ $3,3]$ revealed statistical significance $(p=0,005)$. Thus, these comparative analyses elucidated that plasma OrxA levels of the migraine patients were higher than the levels of control group participants during the non-attack period, while plasma OrxB levels of the migraine patients were higher than the levels of migraine patients during the attack.

Comparison of the plasma OrxA and plasma OrxB levels of the migraine patients (Group 1) during nonattack and attack periods showed that the mean plasma OrxA level was significantly higher during the non-attack period compared with the attack period $(p=0,002)$ (Table 2). On the other hand, the plasma OrxB level was significantly higher during the attack than in the non-attack period $(\mathrm{p}=0,002)$.

Among the 52 patients in Group 1, $36(69,2 \%)$ were diagnosed with migraine without aura, while 16 $(30,8 \%)$ had migraine with aura. The comparison of the mean plasma OrxA and OrxB levels of the patients in Group 1 as per presence or absence of aura revealed no difference between these two subgroups during both attack and non-attack periods (Tables 3 and 4).

Table 1. Plasma OrxA and OrxB levels of the pediatric migraine patients during non-attack and attack periods compared with the levels of healthy controls

\begin{tabular}{|c|c|c|c|c|c|}
\hline & $\begin{array}{l}\text { Control group } \\
(n=46)\end{array}$ & $\begin{array}{l}\text { Study group } \quad(n=52) \\
\text { Non-attack period }\end{array}$ & & $\begin{array}{l}\text { Study group }(n=52) \\
\text { Attack period }\end{array}$ & \\
\hline & $\begin{array}{l}(\mathrm{mean} \pm \mathrm{SD}) \\
\text { [range] }\end{array}$ & $\begin{array}{l}(\text { mean } \pm \text { SD }) \\
\text { [range] }\end{array}$ & $\mathrm{p}$ values & $\begin{array}{l}(\text { mean } \pm \mathrm{SD}) \\
\text { [range] }\end{array}$ & $\mathrm{p}$ values \\
\hline OrxA levels & $\begin{array}{l}503,6 \pm 322 \\
{[19,4-990,3]}\end{array}$ & $\begin{array}{l}639,4 \pm 334,1 \\
{[26,0-1106,4]}\end{array}$ & $p=0,014$ & $\begin{array}{l}476,4 \pm 242,2 \\
{[24,4-780,1]}\end{array}$ & $\mathrm{p}=0,381$ \\
\hline OrxB levels & $\begin{array}{l}1,3 \pm 0,3 \\
{[1,1-3,3]}\end{array}$ & $\begin{array}{l}1,3 \pm 0,2 \\
{[1,1-2,3]}\end{array}$ & $\mathrm{p}=0,287$ & $\begin{array}{l}1,7 \pm 1,5 \\
{[1,1-12,0]}\end{array}$ & $p=0,005$ \\
\hline
\end{tabular}

Table 2. Plasma OrxA and OrxB levels of the pediatric migraine patients during non-attack and attack periods compared with the levels of healthy controls

\begin{tabular}{|l|l|l|l|}
\hline & $\begin{array}{l}\text { Study group }(\mathbf{n}=52) \\
(\text { Non-attack period) }\end{array}$ & $\begin{array}{l}\text { Study group }(\mathbf{n}=52) \\
(\text { Attack period) }\end{array}$ & \\
\hline & $($ mean \pm SD) & (mean \pm SD) & p values \\
\hline Orexin A levels & $639,4 \pm 334,1$ & $476,4 \pm 242,2$ & $\mathbf{p}=\mathbf{0 , 0 0 2}$ \\
\hline Orexin B levels & $1,3 \pm 0,2$ & $1,7 \pm 1,5$ & $\mathbf{p = 0 , 0 0 2}$ \\
\hline
\end{tabular}


Table 3. Comparison of the attack period plasma OrxA and OrxB levels between pediatric migraine patients without and with aura

\begin{tabular}{|l|l|l|l|}
\hline & Migraine without aura (n=36) & Migraine with aura (n=16) & \\
\hline & $($ mean \pm SD) & (mean \pm SD) & p values \\
\hline Plasma OrxA levels & $463,8 \pm 254,1$ & $504,9 \pm 217$ & 0,781 \\
\hline Plasma OrxB levels & $1,8 \pm 1,8$ & $1,5 \pm 0,3$ & 0,422 \\
\hline
\end{tabular}

Table 4. Comparison of the non-attack period plasma OrxA and OrxB levels between pediatric migraine patients without and with aura

\begin{tabular}{|l|l|l|l|}
\hline & Migraine without aura (n=36) & Migraine with aura (n=16) & \\
\hline Plasma OrxA levels & $629,4 \pm 330,8$ & (mean \pm SD) & p values \\
\hline Plasma OrxB levels & $1,3 \pm 0,2$ & $661,9 \pm 351,2$ & 0,481 \\
\hline
\end{tabular}

\section{Discussion}

The orxA (also called hypocretin 1) and orxB (also called hypocretin 2) are both neuropeptides synthesized by the neurons located at the lateral hypothalamic area and dorsomedial hypothalamic nucleus (9). The orexinergic neurons in these locations project into areas such as periaqueductal gray (PAG), nucleus raphe magnus (NRM), and locus ceruleus (LS), which are considered as migraine generators. These areas are rich in terms of orexin receptors: The OX1R (i.e., HCRTR1) is selectively expressed in LC while OX2R (i.e., HCRTR2) is selectively expressed in NRM (11). Holland et al reported that orexins could reduce neurogenic dural vasodilatation and inhibit TVS via activation of OX1R (i.e., anti-nociceptive effects) (12). They could cause neurogenic dural vasodilatation and stimulate TVS via activation of OX2R (i.e., pro-nociceptive effects). It is widely accepted that activation of OX1R leads to analgesic effects via attenuated A- and Cfiber responses to dural stimulation, while activation of OX2R leads to pro-nociceptive effects due to exaggerated $\mathrm{A}-$ and $\mathrm{C}$ - fiber response to dural stimulation $(13,14)$. These findings indicate that activation of OX1R reduces the risk of having a migraine attack, while activation of OX2R increases this risk. Of note, Rainero et al. stated that OX1R had 50-fold more affinity to OrxA than OrxB, while OX2R had similar affinities for both receptors (15).

Conventional migraine medications target the trigeminovascular system; however, Akerman et al. stated that the hypothalamus should be the target for new-generation migraine treatments (16). Hoffmann et al. worked on an experimental animal model and demonstrated that targeting the hypothalamic orexinergic system by a dual orexin receptor antagonist (DORA) DORA-12 could be a potential prophylactic treatment method for migraine (17). In this study, DORA-12 attenuated the neurogenic dural vasodilatation and TVS activation. As such, Cady et al. found in an experimental animal study that dual antagonism of OX1R and OX2R by DORA-12 inhibited trigeminal neural activation (18). It is important to note that DORAs are currently used in treating insomnia, and DORA-12 is the precursor of suvorexant.

Contrary to these findings, Chabi et al., who analyzed the efficacy of filorexant -another DORAin the prophylactic treatment of adult migraine in a randomized, double-blind, placebo-controlled study setting, concluded that this medication was not effective in preventing migraine attacks (19). They gave $10 \mathrm{mg}$ filorexant daily at night-time to their patients with migraine. This dosage selection was based on the fact that the same dose was used to treat insomnia (18-20). It is known that migraine and sleep disorders are closely related to each other (20). Chabi et al. denoted that most of their migraine patients did not have an accompanying sleep disorder (19). They stated that DORAs might be effective in migraine patients who had a concurrent sleep disorder.

In our study, we did not assess our patients in terms of sleep disorders. This fact can be considered as a weakness of our study. On the other hand, it should be noted that our study did not aim to analyze the efficacy of medications in the treatment or prevention of migraine attacks in children. Instead, we investigated the plasma levels of OrxA and OrxB to contribute to the research regarding the role of these neuropeptides in the pathogenesis of pediatric migraine. It is widely accepted that introduction of triptans led to remarkable progress in the acute pharmacologic treatment of migraine (21). However, despite the progress regarding acute treatment 
options, there have been no significant improvements recently in preventive treatment protocols (22).

Ravid et al. reported that orxA inhibited neurogenic dural vasodilatation by reducing the release of calcitonin gene-related peptide (CGRP) from the trigeminal nerve endings (23). It is known that CGRP is a potent vasodilator that is highly effective on dural circulation (24). Moreover, stimulation of the trigeminal ganglion is known to induce secretion of CGRP into the cranial circulation during the initial period of a migraine attack (25). Therefore, current migraine research is focusing on CGRP and orexins for enriching the treatment options.

Sarchielli et al. also investigated the role of OrxA in the pathogenesis of migraine (9). These authors compared the baseline (i.e., non-attack) cerebrospinal fluid (CSF) OrxA levels of 27 adult migraine patients with healthy controls and found that the levels were significantly higher in the former group. They concluded that the increase in the OrxA levels might be due to a hypothalamic response to the stress induced by chronic headaches.

Caproni et al. analyzed the baseline plasma OrxA and OrxB levels in 39 adult patients who were on prophylactic migraine treatment with amitriptyline (10). They reported that plasma OrxA and OrxB levels decreased significantly in the 3rd month of this treatment. These authors stated that their findings were contradictory to the results reported by Sarchielli et al., and they ascribed this difference to the fact that they measured the levels of the orexins in serum while Sarchielli et al. worked on CSF samples $(9,10)$. The analyses of Caproni et al. revealed that pretreatment serum OrxA and OrxB levels of the adult patients with migraine were significantly lower than the levels of healthy controls (10). They concluded that the orexinergic pathway could be defective in patients with migraine.

Since Caproni et al. reported that serum OrxA and OrxB levels of adult migraine patients were significantly lower than those of healthy controls, their findings contradict ours (10). However, it should be considered that our study was conducted with recently diagnosed pediatric migraine patients, while Caproni et al. worked on adult patients (10). We hypothesize that the orexinergic neurons' responsiveness to the 'nociceptive milieu', which can lead to reactive elevation of OrxA levels, might decline by time due to changes in receptor affinities of orexinergic neurons.

As per our knowledge, our study is the first to assess plasma orexin levels in pediatric migraine patients and compare the findings of the attack period with those of the non-attack period. Our results indicated that plasma OrxA levels of pediatric migraine patients analyzed during the non-attack period were significantly higher than those of healthy controls. This finding is in accordance with the antinociceptive effects of OrxA. On the other hand, plasma OrxB levels of the patients measured during the attack period were significantly higher than healthy controls. This result is in line with the pronociceptive effects of OrxB. We also found that plasma OrxA levels were significantly higher during the attack period than the non-attack period. The plasma OrxB levels analyzed during the attack period were significantly higher than plasma OrxB levels measured during the non-attack period. Despite the difference in the analyzed samples, our findings are in line with the results reported by Sarchielli et al., who reported that baseline CSF OrxA levels of migraine patients were higher than those of healthy subjects (9). We postulate that the level of OrxA, which is a neuropeptide with anti-nociceptive effects, is elevated in migraine patients due to nociceptive signals (i.e., reactive elevation) and that migraine attack is associated with elevated OrxB levels. It is known that OrxB is pro-nociceptive, and its dominance may pave the way for a migraine attack. As per our findings, plasma OrxA and OrxB levels did not differ significantly with the presence or absence of aura in pediatric migraine patients. Although these data imply that plasma orexin levels are not directly related to aural symptoms, it is essential to note that the 'potential role of orexins in the pathogenesis of migraine aura' was not the primary focus of this study. On the other hand, our results indicated that the potential relationship between plasma orexin levels and migraine attacks was independent of aura's presence or absence.

\section{Conclusion}

Although it is known that orexins are involved in the pathogenesis of migraine, there are only a few clinical studies analyzing their levels in migraine patients. Our study is the first to analyze the plasma orexin levels in pediatric migraine patients to the best of our knowledge. Our analysis showed that plasma OrxA levels were higher in pediatric migraine patients than healthy controls during the non-attack period, while plasma OrxB levels were higher than healthy controls during attack periods. As per our findings, plasma orexin levels were not directly related to the presence or absence of aura in pediatric migraine patients. We conclude that research focusing on targeting the orexinergic system for improving the preventive treatment of migraine 
should be supported. Our findings indicate that this approach looks promising for the well-being of pediatric migraine patients.

Ethics Committee Approval: This prospective study was approved by the ethical review committee of Ondokuz Mayıs University (OMU) Hospital (2018/174)

Peer-review: Externally peer-reviewed.

Author Contributions:

Concept H. I, O. F. A; Design H.A; Literature search, H. A, Data Collection and Processing H. I, O.F.A, H.A, E.C.C; Analysis or Interpretation, H. I, O.F.A, H.A, E.C.C; Writing H. I, O.F.A, H.A, E.C.C Conflict of Interest: The authors have no interests to declare

Financial Disclosure: This study was funded by Ondokuz May1s University Scientific Research Project Support Fun (PYO.TIP.1901.13.034)

\section{References}

1. Oleson J. The international classification of headache disorders. Cephalalgia. 2004; 24 (suppl):9-160.

2. Winner $\mathrm{P}$, Martinez W, Mate L, Bello L. Classification of pediatric migraine: proposed revisions to the IHS criteria. Headache. 1995;35(7):407-410. doi:10.1111/j.15264610.1995.hed3507407.x

3. Abu-Arefeh I, Russell G. Prevalence of headache and migraine in schoolchildren. BMJ. 1994;309(6957):765-769. doi:10.1136/bmj.309.6957.765

4. Bigal ME, Lipton RB. The epidemiology, burden, and comorbidities of migraine. Neurol Clin. 2009;27(2):321-334. doi:10.1016/j.ncl.2008.11.011

5. Goldstein M, Chen TC. The epidemiology of disabling headache. Adv Neurol. 1982;33:377390.

6. Jain S, Malinowski M, Chopra P, Varshney V, Deer TR. Intrathecal drug delivery for pain management: recent advances and future developments. Expert Opin Drug Deliv. 2019;16(8):815-822. doi:10.1080/17425247.2019.1642870

7. O'Brien HL, Kabbouche MA, Kacperski J, Hershey AD. Treatment of pediatric migraine. Curr Treat Options Neurol. 2015;17(1):326. doi:10.1007/s11940-014-0326-1
8. Tajti J, Szok D, Majláth Z, Tuka B, Csáti A, Vécsei L. Migraine and neuropeptides [published correction appears in Neuropeptides. 2016 Dec;60:91]. Neuropeptides. 2015;52:19-30. doi:10.1016/j.npep.2015.03.006

9. Sarchielli P, Rainero I, Coppola R, Rossi C, Mancini Ml, Pinessi L, et al. Involvement of corticotrophin-releasing factor and orexin- $\mathrm{A}$ in chronic migraine and medication-overuse headache: findings from cerebrospinal fluid. Cephalalgia. 2008;28(7):714-722. doi:10.1111/j.1468-2982.2008.01566.x

10.Caproni S, Corbelli I, Pini LA, Cupini ML, Calabresi P, Sarchielli P. Migraine preventive drug-induced weight gain may be mediated by effects on hypothalamic peptides: the results of a pilot study. Cephalalgia. 2011;31(5):543-549. doi:10.1177/0333102410392605

11.Holland P, Goadsby PJ. The hypothalamic orexinergic system: pain and primary headaches. Headache. 2007;47(6):951-962. doi:10.1111/j.1526-4610.2007.00842.x

12.Holland PR, Akerman S, Goadsby PJ. Orexin 1 receptor activation attenuates neurogenic dural vasodilation in an animal model of trigeminovascular nociception. J Pharmacol Exp Ther. 2005;315(3):1380-1385. doi:10.1124/jpet.105.090951

13.Bartsch T, Levy MJ, Knight YE, Goadsby PJ. Differential modulation of nociceptive dural input to [hypocretin] orexin A and B receptor activation in the posterior hypothalamic area. Pain. 2004;109(3):367-378. doi:10.1016/j.pain.2004.02.005

14. Holland PR, Akerman S, Goadsby PJ. Modulation of nociceptive dural input to the trigeminal nucleus caudalis via activation of the orexin 1 receptor in the rat. Eur $J$ Neurosci. 2006;24(10):2825-2833. doi:10.1111/j.14609568.2006.05168.x

15.Rainero I, Rubino E, Gallone S, Fenoglio P, Picci LR, Giobbe L, et al. Evidence for an association between migraine and the hypocretin receptor 1 gene. J Headache Pain. 2011;12(2):193-199. doi:10.1007/s10194-011-0314-8

16. Akerman S, Holland PR, Goadsby PJ. Diencephalic and brainstem mechanisms in migraine. Nat Rev Neurosci. 2011;12(10):570584. Published 2011 Sep 20. doi:10.1038/nrn3057

17.Hoffmann J, Supronsinchai W, Akerman S, Andreou AP, Winrow CJ, Renger $\mathrm{J}$, et al. Evidence for orexinergic mechanisms in migraine. Neurobiol Dis. 2015;74:137-143. doi:10.1016/j.nbd.2014.10.022 
18. Cady RJ, Denson JE, Sullivan LQ, Durham PL. Dual orexin receptor antagonist 12 inhibits expression of proteins in neurons and glia implicated in peripheral and central sensitization. Neuroscience.

2014;269:79-92. doi:10.1016/j.neuroscience.2014.03.043

19.Chabi A, Zhang Y, Jackson S, Cady R, Lines C, Herring WJ, et al. Randomized controlled trial of the orexin receptor antagonist filorexant for migraine prophylaxis. Cephalalgia. 2015;35(5):379-388. doi: $10.1177 / 0333102414544979$

20.Bigal ME, Hargreaves RJ. Why does sleep stop migraine? [published correction appears in Curr Pain Headache Rep. 2014 Jan;18(1):390]. Curr Pain Headache Rep. 2013;17(10):369. doi:10.1007/s11916-013-0369-0

21.Ferrari MD, Roon KI, Lipton RB, Goadsby PJ. Oral triptans (serotonin 5-HT(1B/1D) agonists) in acute migraine treatment: a meta-analysis of 53 trials. Lancet. 2001;358(9294):1668-1675. doi:10.1016/S0140-6736(01)06711-3

22.Lipton RB, Bigal ME, Diamond M, Freitag F, Reed ML, Stewart WF. et al. Migraine prevalence, disease burden, and the need for preventive therapy. Neurology. 2007;68(5):343-349. doi:10.1212/01.wnl.0000252808.97649.21

23.Ravid S. Migraine \& paediatric obesity: a plausible link?. Indian J Med Res. 2014;139(3):343-348.

24.Jansen-Olesen I, Mortensen A, Edvinsson L. Calcitonin gene-related peptide is released from capsaicin-sensitive nerve fibres and induces vasodilatation of human cerebral arteries concomitant with activation of adenylyl cyclase. Cephalalgia. 1996;16(5):310-316. doi:10.1046/j.1468-2982.1996.1605310.x
25.Goadsby PJ, Edvinsson L, Ekman R. Release of vasoactive peptides in the extracerebral circulation of humans and the cat during activation of the trigeminovascular system. Ann Neurol. 1988;23(2):193-196. doi:10.1002/ana.410230214 